\title{
De lo local a lo global en la lucha por la democratización de las comunicaciones
}

From the local to the global in the struggle for the democratization of communications

Do local ao global na luta pela democratização das comunicações

Larisa KEJVAL, Argentina

Universidad de Buenos Aires / larisakej@yahoo.com.ar

Chasqui. Revista Latinoamericana de Comunicación

N. ${ }^{\circ} 140$, abril - julio 2019 (Sección Monográfico, pp. 75-94)

ISSN 1390-1079 / e-ISSN 1390-924X

Ecuador: CIESPAL

Recibido: 25-02-2019/Aprobado: 10-07-2019 


\section{Resumen}

El artículo describe y analiza la estrategia a escala global que el movimiento de radios comunitarias, populares y alternativas latinoamericano desplegó, a partir de los años noventa y durante la primera década de este siglo, con el propósito de incidir en los estándares internacionales en materia de derecho a la libertad de expresión y a la información, como camino para la democratización de las comunicaciones en un contexto de creciente globalización. Para eso se recupera la literatura producida, fundamentalmente, por las redes latinoamericanas ALER y AMARC ALC. Esta revisión histórica puede resultar inspiradora de cara a los nuevos desafíos que plantea la lucha por el derecho a la comunicación en los contextos actuales.

Palabras clave: comunicación comunitaria; comunicación popular; comunicación alternativa; radio; políticas de comunicación; derecho a la comunicación

\section{Abstract}

The article describes and analyzes the global strategy that the Latin American movement of community, popular and alternative radio deployed.From the nineties and during the first decade of this century, with the purpose of influencing international standards, regarding the right to freedom of expression and information, as a way to democratize communications in a context of increasing globalization. For that matter, it recovers the literature produced, fundamentally, by the Latin American networks ALER and AMARC ALC. This historical review can be inspiring facing - the new challenges posed by the struggle for the right to communication in current contexts.

Keywords: community communication; popular communication; alternative communication; radio; communication policies; right to communication

\section{Resumo}

$\mathrm{O}$ artigo descreve e analisa a estratégia em escala global que o movimento de rádio comunitária, popular e alternativa latino-americana implantou, a partir dos anos noventa e durante a primeira década deste século, com o objetivo de influenciar os padrões internacionais no campo da direito à liberdade de expressão e informação, como forma de democratizar as comunicações em um contexto de crescente globalização. Para isso, o artigo recupera a literatura produzida, fundamentalmente, pelas redes latino-americanas ALER e AMARC ALC. Esta revisão histórica pode ser inspiradora diante dos novos desafios colocados pela luta pelo direito à comunicação nos contextos atuais.

Palavras-chave: comunicação comunitária; comunicação popular; comunicação alternativa; rádio; políticas de comunicação; direito à comunicação 


\section{Introducción}

Elpresente artículo se propone presentar uno delos resultados delainvestigación desarrollada en el marco de la propia tesis doctoral: la constatación de que el movimiento de radios comunitarias, populares y alternativas latinoamericano se dio, a partir de los años noventa y durante la primera década del nuevo siglo, no sólo diversas estrategias de incidencia en los territorios locales sino también, en un contexto de creciente globalización, una estrategia deliberada, coherente, sistemática y progresiva de incidencia a escala global para la democratización de las comunicaciones. La propuesta de las próximas líneas es describir y analizar sintéticamente en qué consistió esta estrategia de incidencia global de tal modo de que sirva de punto de referencia para los desafíos a encarar en el presente. Pero antes avanzar en este propósito, primeramente explicitaremos desde qué marco investigativo -con su respectivo enfoque teórico y metodológico- hemos llegado a la constatación en la que luego nos detendremos.

La investigación desarrollada interrogó acerca de la identidad política colectiva de las radios comunitarias, populares y alternativas argentinas entre su surgimiento a mediados de los años ochenta hasta diciembre de $2015^{1}$. En principio, las emisoras de este tipo son gestionadas por organizaciones o colectivos que no persiguen el fin de lucro. Asimismo, si bien realizan sus acciones desde distintas orientaciones políticas o priorizan problemas diferentes, comparten el horizonte de contribuir a procesos de resistencia o de transformación del orden comunicacional y social en pos de sociedades más justas e igualitarias. No obstante, las nociones de radio comunitaria, de radio popular y de radio alternativa también están atravesadas por debates y diferencias. A partir de aquí, algunos de mis trabajos han estado orientados por la pregunta acerca de las significaciones ligadas a estas nociones. $O$, lo que es lo mismo, desde nuestra perspectiva teórica, por la pregunta sobre la identidad política colectiva. Cuando nos referimos a la identidad política de las radios estamos aludiendo, también, a lo que algunos autores han denominado proyectos político culturales (Villamayor y Lamas, 1998) y político comunicativos (ALER, 2007 y ALER y AMARC ALC, 2009).

Aquella indagación se inscribió en el campo académico de la comunicación comunitaria, popular y alternativa. Complementariamente, la concepción de identidad política colectiva recuperó los aportes del análisis político del discurso iniciado por Laclau y Mouffe (Laclau y Mouffe, 2010; Laclau, 2011). En este sentido, compartimos con otras investigaciones recientes la búsqueda por contribuir a la conformación de este campo desde el entrecruzamiento con perspectivas teóricas que hasta el momento habían resultado ajenas.

Desde mediados de los años ochenta en adelante, las radios comunitarias, populares y alternativas argentinas se fueron encontrando y articulando en

1 Una versión completa de este trabajo ha sido publicado en formato libro (Autora, año). 
redes nacionales o latinoamericanas. A nivel nacional: la Asociación de Radios Comunitarias (ARCO) que funcionó entre 1987 y 1989; el Foro Argentino de Radios Comunitarias (FARCO), sucesor de aquella primera red a partir de la década de 1990; la Red Nacional de Medios Alternativos (RNMA) creada en el año 2004; el capítulo nacional de la Asociación Mundial de Radios Comunitarias (AMARC-Argentina) a partir de 2008; y la Red Colmena fundada en 2014. A nivel latinoamericano: la Asociación Latinoamericana de Educación Radiofónica (ALER) creada en 1972 y la misma AMARC en su regional América Latina y Caribe, fundada en 1990. Asimismo, desde la década de 1980 centros de comunicación/ educación nacionales han promovido el desarrollo y fortalecimiento de las emisoras. Nos referimos, por ejemplo, al rol desempeñado por el Instituto de Cultura Popular (INCUPO), el Centro de Comunicación La Crujía, el Centro Nueva Tierra y, ya en el nuevo siglo, el colectivo de educación popular Abrojos y el Centro de Producciones Radiofónicas (CPR). A estos aportes se agregan las iniciativas de intelectuales del campo académico estrechamente comprometidos con el fortalecimiento de la comunicación comunitaria, popular y alternativa, tanto en nuestro país como en el conjunto de América Latina. Estas redes, centros de comunicación/educación e intelectuales produjeron un gran número de publicaciones con el propósito de visibilizar las experiencias de las radios en el espacio público, con el objetivo de multiplicar aprendizajes para contribuir a su fortalecimiento, o bien, para reflexionar y problematizar sobre sus prácticas y horizontes. A ellas se suman, fundamentalmente a partir de los años noventa, las publicaciones producidas por las propias emisoras argentinas con el propósito de dar a conocer sus historias, sistematizar y compartir sus saberes, y expresar las perspectivas político comunicacionales en las que sustentan sus proyectos. En cada una de estas publicaciones emergen rasgos de la identidad política o, dicho en otros términos, de los proyectos político comunicacionales que éstas encarnan. En síntesis, la superficie discursiva a la que nos acercamos para aproximarnos a la pregunta por la identidad política de las emisoras estuvo conformada por un corpus conformado por 84 publicaciones referidas a las radios comunitarias, populares y alternativas argentinas y latinoamericanas, producidos por las mismas emisoras, por las redes en las que se nuclearon y por centros de comunicación/educación e intelectuales del campo académico. Entre estas publicaciones podemos distinguir libros, cuadernillos, artículos de libros, artículos de revistas académicas y declaraciones. En el caso de los artículos, la mayoría fueron publicados en revistas académicas nacionales o latinoamericanas como Causas y Azares, Oficios Terrestres, Diálogos de la Comunicación y la propia Chasqui.

Desde la perspectiva del análisis político del discurso, en el abordaje de este corpus procuramos identificar las huellas del proceso de constitución de la identidad política colectiva de las radios comunitarias, populares y alternativas, entendida como proceso de configuración discursiva. Para eso centramos la atención en identificar: 
- Las condiciones de producción que incidieron en la emergencia y en la constitución de la identidad política de las emisoras.

- Las relaciones de antagonismo que resultaron constitutivas de la identidad política de las radios y ante las cuales se configuraron relaciones de equivalencia.

- Los principales significantes que operaron como puntos nodales, y eventualmente como significantes vacíos, capaces de condensar la identidad de las radios comunitarias, populares y alternativas y, en este sentido, una pluralidad de significados.

- Los significados y demandas articulados en términos de regularidades a esos significantes. $\mathrm{O}$, dicho en otros términos, las huellas de la lógica de la equivalencia en la constitución de la identidad política.

- Los significados y demandas que permanecieron como momentos diferenciales. - Dicho en otras palabras, las huellas de la lógica de la diferencia en el proceso de configuración de la identidad política de las emisoras.

- Las continuidades y desplazamientos en este conjunto de relaciones constitutivas de la identidad política de las radios comunitarias, populares y alternativas a lo largo de la historia.

A partir de la identificación de estas huellas desarrollamos un análisis basado en el ejercicio interpretativo.

Coincidimos con Gerbaldo (2014, p. 14) cuando sostiene que "las radios comunitarias en Argentina fueron desarrollándose desde los 80 a la actualidad al calor de las redes de radios a nivel latinoamericano o internacional (ALER y AMARC-ALC) que las nucleaban y con las que se identificaban". Como consecuencia, y tal como hemos anticipado, nuestro análisis no sólo se centró en un corpus de publicaciones nacionales sino que se amplió en incluyó materiales publicados por redes, centros de comunicación/educación y revistas de alcance latinoamericano. Es, precisamente, la sistematización y el análisis de este tipo de publicaciones a lo largo de nuestra investigación, lo que nos permitió llegar a la constatación que presentaremos a continuación ${ }^{2}$ : el movimiento de radios comunitarias, populares y alternativas latinoamericanas se dio, a partir de los años noventa en adelante, no sólo diversas estrategias de incidencia en los territorios locales sino también una estrategia deliberada, coherente, sistemática y progresiva de incidencia a escala global en pos de la democratización de las comunicaciones.

2 El trabajo de investigación permitió llegar a otras conclusiones en el proceso de constitución de la identidad política de las emisoras argentinas, tal como puede leerse en trabajos previos. Particularmente aqui nos detendremos en el análisis de la estrategia de incidencia global que se dio el movimiento a nivel latinoamericano para incidir en los estándares internacionales en materia de derecho a la libertad de expresión y a la información, tarea que no hemos encarado con anterioridad. 


\section{Los años noventa: de la resistencia a la incidencia}

La investigación recién sintetizada nos permitió comprender que en el contexto de la extensa década neoliberal -iniciada a fines de los años ochenta y puesta en crisis a inicios del nuevo siglo- las radios comunitarias, populares y alternativas latinoamericanas se propusieron, como una de sus principales tareas políticas, resistir al neoliberalismo. Frente al imperio del mercado como escenario de las relaciones sociales; a la despolitización de los sujetos, sus prácticas y sus cuerpos; al fin de las ideologías y la historia; resistir al neoliberalismo supuso politizar lo despolitizado. Esto significó asumir la tarea de tematizar el poder en todos los ámbitos. De lo que se trataba era de poner en argumento aquello que se presentaba como natural, como sentido común, para poner nombre a procesos históricos, a decisiones políticas, a relaciones de poder. Al despolitizar lo despolitizado se abría la posibilidad de lo que se presentaba como inevitable pudiera ser de otra manera. De este modo se criticaba la idea imperante de un presente perpetuo y se recuperaba la resquebrajada idea de otro futuro posible.

Asimismo, frente a la creciente centralidad de los medios de comunicación en la cultura y en la sociedad, durante los años noventa uno de los fundamentos de las radios comunitarias, populares y alternativas se basó en la explícita decisión de intervenir políticamente desde el territorio de los medios de comunicación audiovisuales. ALER lo sintetizó de la siguiente forma (1996, p. 31): "Estos nuevos rasgos de la comunicación masiva indican que los medios se han convertido en un campo estratégico de acción”. Si la centralidad de los medios se manifestaba, entre otras cosas, en su capacidad para visibilizar y legitimar ideas, sujetos y acontecimientos, una de las tareas que las radios comunitarias, populares y alternativas asumieron para sí, fue la de constituirse en espacio de expresión y fortalecimiento de sectores e ideas no hegemónicos.

Del mismo modo, durante aquellos años las emisoras se propusieron reconstruir los lazos sociales erosionados y articular las resistencias que se presentaban fragmentadas y dispersas. El terrorismo de Estado impuesto por las dictaduras militares latinoamericanas ya había dejado como saldo la desarticulación de los lazos sociales y políticos de sindicatos, agrupaciones políticas, grupos eclesiales inspirados en la Teología de la Liberación, movimientos estudiantiles y organizaciones territoriales. En la década de 1990, a la desestructuración operada por la dictadura militar se sumó la fuerza individualizadora característica del capitalismo en su fase neoliberal. En este contexto resistir al neoliberalismo significó crear espacios comunicacionales que permitieran reconstruir lazos sociales y articular múltiples disputas. Así lo expresaba Mata (1993, p. 7):

Hoy las emisoras asumen más plenamente su papel mediador. Comprenden que la expresión de los diversos sectores debe servir para su mutuo reconocimiento, para que dialoguen entre sí en pos de estrategias globales de acción. En algunos casos 
las emisoras buscan vincular diferentes espacios regionales. En otros fomentan el reconocimiento de las múltiples etnias o nacionalidades que coexisten en un mismo país. En muchos se proponen contribuir al necesario diálogo entre hombres y mujeres, jóvenes y adultos.

La autora caracterizó a las emisoras latinoamericanas como medios de rearticulación del campo popular fragmentado y debilitado.

La decisión de resistir la hegemonía del capitalismo neoliberal en tanto modelo económico, político, social y cultural podría haber reducido los proyectos político- comunicacionales de las radios que nos ocupan a la construcción de refugios expresivos o de pequeñas comunidades de intereses. No obstante, además de resistir las emisoras expresaron la intención de lograr incidencia en la transformación de la sociedad. Así lo expresaba ALER (1996, p. 70): "Seguras de su papel como actores sociales y para incidir en la sociedad, son radios que salen de la marginalidad y se proyectan más allá de su ámbito geográfico y sectorial de funcionamiento".

Desde la misma red, Geerts y Van Oeyen (2001, p. 43) definieron la incidencia de una radio como:

el peso de su presencia y de su influencia en la sociedad, los procesos que genera, la mella que hace para provocar un cambio a favor o en contra de algo, la capacidad de la radio para levantar polvo. Una radio que incide, deja una marca, es una radio protagonista que no se puede obviar.

En primer lugar, incidencia significó la búsqueda de masividad, entendida como la construcción de audiencias numerosas y diversas, de tal modo que no quedaran reducidas a las organizaciones del campo popular (López Vigil, 1997), a los ya concientizados (Mata, 1994), a los convencidos (Lamas y Lewin, 1995), al partido (Galavotti y Randazzo, 2001); es decir, a quienes compartían una mirada reflexiva y crítica similar a la de quienes producían las radios. Para las asociaciones de radios latinoamericanas $\mathrm{ALER}^{3}$ y AMARC ALC la pregunta por la masividad y la popularidad significó poner en relación los objetivos sociales y políticos transformadores con el reconocimiento de las características del sistema de medios de comunicación imperante y con los modos en que ese sistema configura su- público-. En este sentido ALER (1996, p. 72 y 73) afirmaba:

Las radios populares son medios masivos y, como tales, forman parte de un mercado de comunicación poblado por otras ofertas. Es un mercado regido por normas implacables que ha tenido la capacidad de modelar culturalmente a los sujetos como públicos de una cultura masiva. Este reconocimiento implica tener en cuenta que las audiencias no son libres y que están habituadas a

3 La pregunta por las audiencias llevó a ALER a desarrollar una línea de acción vinculada a la investigación de las programaciones y las audiencias de las emisoras. 
códigos, géneros, modos de interactuar con las demás radios que no pueden ser desconocidas a la hora de planificar estrategias alternativas.

Desde AMARC ALC López Vigil (1996, p. 26) lo expresó en estos términos: “Si apostamos por un medio de comunicación masivo es para influir en la opinión pública, para contribuir a mejorar la sociedad".

La pregunta por las audiencias conllevó la atención hacia otras cuestiones: la utilización de géneros y formatos reconocibles por los públicos de los medios masivos, la apertura de los criterios estéticos a los gustos y repertorios populares -incluso a pesar de que fueran moldeados por el mercado-, la inclusión del entretenimiento y de lo lúdico en las producciones, la opinión bien argumentada, la interpelación a la sensibilidad y no sólo a la reflexión racional, la formación y la capacitación de comunicadoras y comunicadores, la calidad técnica de las producciones, la inserción en el mapa de medios local y la pregunta por las audiencias.

\section{La estrategia de incidencia a escala global}

La voluntad de incidir, que el movimiento latinoamericano de radios comunitarias asumió para sí durante los años noventa, también significó la explícita voluntad de profundizar la articulación en red. Si en las décadas precedentes la conformación de redes latinoamericanas de radios había estado orientada, principalmente, por el intercambio de experiencias y el fortalecimiento de las emisoras a través de la capacitación, a lo largo de los años noventa puede leerse una mayor interpelación a conformar redes de emisoras nacionales y continentales con los propósitos de aumentar la potencia política y comunicacional de las radios y de ampliar sus alcances más allá de los límites de inserción locales en un contexto de globalización. Por ejemplo, desde ALER, Gutiérrez (1997, p. 32) sostenía: “aunque está claro que las radios se deberán a sus audiencias locales y es allí donde encuentran mayoritariamente su razón de ser, su propuesta trascenderá las fronteras de su zona de cobertura, por razones políticas y por razones prácticas”. América Latina en Red (ALRED) inaugurada en 1997 fue la estrategia que desarrolló ALER a través de la tecnología satelital "para construir una red continental que inter-comunique a las emisoras populares y a la vez posibilite la globalización de su propuesta al conjunto de las sociedades latinoamericanas" (Gutiérrez, 1997, p. 32). A su vez, en 1996 AMARC ALC fundó la Agencia Informativa Púlsar, un servicio informativo regional a través de Internet producido con los aportes de diferentes emisoras de la región. Según Girard (1997, p. 27), su primer director, "las mismas tecnologías de la comunicación que hacen posible la globalización y la centralización de la programación, también hacen posible que estas radios ciudadanas trabajen con la sociedad civil en su intento por influir en la aldea mundial". 
La voluntad de incidencia y la consecuente articulación en red no sólo impactaron en las programaciones de las emisoras. También, inauguraron un proceso de confluencia de redes regionales con el propósito de acordar una política deliberada, coherente, sistemática y progresiva a escala global para la incidencia en los estándares internacionales en materia de derecho a libertad de expresión y a la información, entendidos como marcos interpretativos de estos derechos, en pos de democratizar las legislaciones latinoamericanas en materia de radiodifusión que vedaban y limitaban la legalidad de las radios comunitarias, populares y alternativas. El acontecimiento inaugural en este sentido fue el Seminario "Democratizar el Espectro Radioeléctrico" que en noviembre de 1996 reunió en Pozo de Rosas, Venezuela, a coordinadoras nacionales y representantes de medios comunitarios de Argentina, Brasil, Chile, Colombia, Ecuador, El Salvador, Guatemala, México, Paraguay, Perú, Uruguay y Venezuela; a abogados provenientes de varios países de la región; y a directivos de diez redes y organismos internacionales: ALER, AMARC, el Centro Internacional de Estudios Superiores de Comunicación para América Latina (CIESPAL), la Federación Latinoamericana de Facultades de Comunicación Social (FELAFACS), la Federación Internacional de Periodistas (FIP), PROA, Radio Nederland Training Centre (RNTC), SCC, VIDEAZIMUT y World Association for Christian Communication (WACC). En la declaración conjunta de este evento se comprendió, por primera vez, a la radiodifusión "como el ejercicio del derecho humano básico de expresión e información a través de un soporte tecnológico". Asimismo, desde esta perspectiva, se denunció la limitación al derecho de antena que afectaba a las radios comunitarias, populares y alternativas:

Entendemos la libertad de expresión e información y su ejercicio como fundamento esencial de la democracia. Esto implica promover acciones jurídicas y marcos regulatorios que reconozcan y aseguren la democratización del espectro radioeléctrico, el acceso y participación sin discriminaciones para todos los sectores (estatal, comercial y no comercial), la transparencia en la información sobre la gestión y administración del espectro y condiciones técnicas razonables para el otorgamiento y operación de los servicios de radio y televisión.

El número 56 de la Revista Chasqui, editado en diciembre de 1996, publicó esta declaración y algunas de las intervenciones del seminario. Allí Roncagliolo (1996, p. 50) enfatizó: "Toda nuestra reflexión jurídica, por lo tanto, va a basarse en el derecho humano a las comunicaciones". Este encuentro, como su declaración final, dieron inicio a una estrategia internacional de incidencia, motorizada por las redes de radios junto con otros actores, que cobrará mayor impulso en los años siguientes y que fue de vital importancia para impulsar nuevos marcos legales en la región.

Esta estrategia, iniciada en los años noventa y sostenida durante la primera década del nuevo siglo, se basó en un accionar que comprendió: 
- un inédito activismo en torno al derecho a la comunicación y la democratización de las comunicaciones a escala mundial que articuló a expertos y militantes sociales y que se expresó en diversos foros, cumbres y campañas.

- la sistematización y la investigación de marcos normativos, políticas y prácticas en toda la región, e incluso en otros continentes, como sustento para elaborar principios y recomendaciones que permitieran ampliar y precisar los alcances de los derechos a la información y a la libertad de expresión y para identificar los casos de vulneración de estos derechos humanos.

- la articulación con investigadores, organismos internacionales y organizaciones de la sociedad civil que comparten el propósito de democratizar las comunicaciones en el conjunto de la región para complementar esfuerzos y conformar fuerzas desde la sociedad civil capaces de ejercer incidencia.

- un trabajo sostenido para incidir en la elaboración de estándares internacionales entendidos como marcos de comprensión del derecho a la libertad de expresión y a la información, con especial atención a la Comisión Interamericana de Derechos Humanos y su Relatoría para la Libertad de Expresión y a la Relatoría especial de Naciones Unidas sobre libertad de opinión y de expresión.

- un trabajo de incidencia para que los Estados asuman dichos estándares internacionales como orientación de sus propias legislaciones, políticas y prácticas, explicitando la supremacía de los tratados y declaraciones internacionales firmadas por los propios Estados.

- la explícita denuncia pública de los Estados cuando sus normativas, políticas y prácticas vulneraron dichos estándares internacionales en materia de libertad de expresión y derecho a la información. Esto supuso el seguimiento atento de las prácticas discrecionales y discriminatorias en la adjudicación, renovación y revocación de frecuencias como la subasta económica; el establecimiento de barreras al acceso equitativo de las comunidades indígenas, organizaciones sociales y otros medios no comerciales a la radio y la TV; y la renovación automática de concesiones, entre otras prácticas.

Asimismo, esta estrategia articulada se sostuvo en un entramado conceptual que, en un ejercicio interpretativo, procuraremos sintetizar en las siguientes líneas. La democratización de las comunicaciones, y con ello la democratización de las sociedades, constituyeron los principales horizontes que orientaron a esta política. Los derechos humanos, más particularmente el derecho a la libertad de expresión y el derecho a la información en sus más amplios sentidos, fueron los pilares del marco conceptual de esta política. De acuerdo a las Declaraciones de las Relatorías sobre Libertad de Expresión de la CIDH y de las Naciones Unidas, 
el alcance del derecho a la libertad de expresión comprende a la radiodifusión y los demás servicios de comunicación audiovisual como formas o vías para su ejercicio. Asimismo, las nociones de diversidad y de pluralismo constituyen los principales objetivos y fundamentos en los cuales debe apoyarse la regulación de los servicios de comunicación audiovisual y las políticas de comunicación. Procurar diversidad y pluralismo en el sistema de medios se torna relevante si se los considera como uno de los espacios privilegiados donde se libran las disputas por los sentidos que conforman las identidades y la percepción de la realidad social y política; como uno de los escenarios fundamentales donde se legitiman ideas, sujetos y los asuntos públicos a ser debatidos y atendidos. En este sentido, los marcos regulatorios deben explicitar el reconocimiento de tres diferentes sectores: público, comercial y social/sin fines de lucro, el cual incluye los medios comunitarios. Todos ellos deben ser capaces de operar en, y tener acceso equitativo a, todas las plataformas de transmisión disponibles. Las medidas específicas para promover la diversidad pueden incluir reservas de frecuencias para distintos tipos de medios. Los procesos de concentración de la propiedad, de transnacionalización y de globalización del mercado de bienes simbólicos vividos en las últimas décadas, así como la histórica concentración geográfica de la producción comunicacional en las grandes ciudades de la región y el mundo, constituyen una amenaza, cuando no directamente la desaparición, de la pluralidad de expresiones culturales e informativas. Por último, los procesos de transición digital son considerados, potencialmente, como una oportunidad. No obstante, son también considerados una amenaza para la diversidad y el pluralismo cada vez que quedan al margen de las regulaciones en materia de servicios de comunicación audiovisual, no son sujetos a debate social o se determinan discrecionalmente.

Esta estrategia facilitó la emergencia de la sociedad civil como actor social y político -articulado en redes, foros y campañas- en la lucha mundial por la democratización de las comunicaciones, la revitalización de los espacios de debate acerca de las políticas de comunicación y el surgimiento de un inédito activismo comunicacional de carácter global. Para Néstor Busso (2011, p. 26) estos espacios "han permitido catalizar procesos de convergencia de la sociedad civil, con repercusiones que van más allá de los eventos mismos, dando lugar a articulaciones más duraderas". Estas articulaciones se expresaron, por el ejemplo, en el Foro Social Mundial a partir de 2001, en la campaña CRIS (Communication Rights in the Information Society) en el contexto de la Cumbre Mundial de la Sociedad de la Información ${ }^{4}$ y en la Cumbre de los Pueblos desarrollada paralelamente a la Cumbre de las Américas en 2005. Asimismo, Mattelart (2007, p. 30) dio cuenta de un período de veinte años en los que las discusiones internacionales acerca de la democratización de las comunicaciones atravesaron un período de "glaciación". La retirada de Gran Bretaña y Estados

4 Sintéticamente, la campaña promovió la idea de que el derecho a la comunicación y los derechos humanos en su conjunto deben constituir el fundamento de la Sociedad de la Información. 
Unidos de la UNESCO tras la presentación del Informe Mac Bride y la propuesta del Nuevo Orden Mundial de la Información y Comunicación (NOMIC), dio inicio a un período de congelamiento de los debates que, según el mismo autor, se extendió hasta 2004 cuando en ese organismo comenzaron las negociaciones acerca de la Convención Internacional sobre la Protección y Promoción de la Diversidad de las Expresiones Culturales. Esos veinte años coincidieron, de acuerdo con Mattelart (2007, p. 30), con "el desmantelamiento de las regulaciones públicas y la penetración de marcos jurídicos favorables al avance del espacio de la racionalidad mercantil -la impropiamente llamada desregulación". A las discusiones que resurgieron en el seno de la UNESCO a partir de 2004, Mattelart sumó las acontecidas a propósito de la Cumbre Mundial de la Sociedad de la Información donde, por primera vez en la historia del sistema de Naciones Unidas, las organizaciones no gubernamentales fueron invitadas a dar su opinión en el transcurso de las conferencias preparatorias. Según el autor, a pesar de la heterogeneidad de sus componentes, y sin llegar a limar asperezas, estas organizaciones lograron expresarse con una sola voz cuando se trataba de afirmar los principios del derecho a la comunicación. Paralelamente, en el Foro Social Mundial -que desde 2001 en adelante reunió a movimientos sociales, organizaciones y activistas de todo el mundo en torno a estrategias globales de resistencia y de construcción de alternativas al capitalismo neoliberal- los temas de comunicación y cultura fueron ocupando un lugar crecientemente estratégico. En todos estos debates Mattelart reconoció la acumulación de las redes internacionales que fueron estableciendo relaciones entre sí, como en el caso del frente comunicacional que en América Latina agrupó a organizaciones como AMARC, ALER, la Agencia Latinoamericana de Información (ALAI), WACC y la Organización Católica Latinoamericana y Caribeña de Comunicación (OCLACC), entre otras.

En el particular caso de AMARC ALC la estrategia que venimos sintetizando quedó expresada en una serie de publicaciones producidas por la red regional a través de su Programa de Legislaciones y Derecho a la Comunicación. En 2008 la asociación concluyó el proceso de elaboración de los "Principios para un marco regulatorio democrático sobre radio y TV comunitaria”. Se trata de 14 principios propuestos como guía para la elaboración, aprobación e implementación de regulaciones sobre radiodifusión comunitaria que sean compatibles con los estándares internacionales de derechos humanos. El documento fue el resultado de una investigación realizada en 29 países de los cinco continentes que se propuso identificar las normativas y las políticas públicas dirigidas a reconocer y a promover la radio y la TV comunitarias. Como estrategia tendiente a validar este insumo, en su proceso de elaboración y revisión estuvieron involucrados decenas de investigadores, miembros de emisoras comunitarias y autoridades de la red mundial. Los "principios" fueron presentados ante la Comisión Interamericana de Derechos Humanos (CIDH) y su Relatoría Especial para la Libertad de Expresión en octubre de 
2008. Más adelante, ya en 2010, el Relator Especial de Libertad de Opinión de Naciones Unidas, Frank La Rue, presentó ante el Consejo de Derechos Humanos del organismo su II informe sobre la situación de la libertad de expresión en el mundo. En el capítulo de conclusiones y recomendaciones de este informe, la relatoría reconoció a los medios de comunicación comunitarios y recomendó a los Estados establecer marcos legales que reconocieran y regularan la comunicación comunitaria dentro del marco de los 14 principios propuestos por AMARC, estableciendo un balance equitativo entre los medios comunitarios, los comerciales y los públicos o estatales. Por otra parte, bajo la coordinación de Gómez y Aguerre, AMARC ALC publicó en 2009 "Las mordazas invisibles". Basado en un trabajo de investigación realizado durante la segunda mitad de 2008, la publicación analizó la gestión del espectro radioeléctrico en materia de radio y televisión en varios países de América Latina con el propósito de determinar las barreras que obstaculizan el efectivo cumplimiento del derecho a la información y a la libertad de expresión. AMARC ALC fue más allá de los marcos normativos para identificar otros obstáculos en la generación de un entorno mediático diverso y plural. Más de 15 investigadores e investigadoras de toda la región estuvieron comprometidos en el relevamiento y análisis de la situación en la región. Este extenso trabajo fue proyectado explícitamente como un documento de referencia para los distintos organismos del Sistema Interamericano de Derechos Humanos y para las organizaciones que trabajan en la defensa y promoción de la libertad de expresión. Más tarde, en 2010 AMARC ALC publicó los "Principios para garantizar la diversidad y el pluralismo en la radiodifusión y los servicios de comunicación audiovisual". Se trata de 40 principios orientados a servir de base para la definición e implementación de políticas, leyes, reglamentaciones y prácticas de radiodifusión. Este trabajo surgió en un contexto en el que diversos países del continente comenzaron a vivenciar intensos debates por nuevas normativas en la materia. En este trabajo AMARC ALC no sólo orientó las recomendaciones a garantizar plenos derechos para las emisoras comunitarias, sino que extendió su labor en pos de la diversidad y el pluralismo en el conjunto de los servicios de comunicación audiovisual. Loreti y Gómez fueron los autores de estos 40 principios. Siguiendo la misma línea de las publicaciones anteriores, su elaboración fue enriquecida gracias al aporte de un conjunto de organismos internacionales y organizaciones que trabajan en la promoción de la comunicación como derecho humano. Por último, durante dos períodos consecutivos -2009 y 2010- AMARC ALC publicó el "Informe Anual sobre Diversidad y Pluralismo en la Radiodifusión". Tomando como marcos conceptuales las producciones recién mencionadas, así como los tratados y declaraciones internacionales sobre libertad de expresión y derecho a la información, a través de estos informes la red realizó un relevamiento y un seguimiento de las principales barreras que impiden la diversidad y el pluralismo en la radiodifusión en 14 países de América Latina y 
el Caribe, así como de las transformaciones en los marcos normativos y en las políticas ligadas a la radiodifusión.

La política que venimos sintetizando tuvo como resultado el reconocimiento de la existencia de los medios comunitarios en diversas declaraciones internacionales. En su declaración conjunta realizada a fines de 2001, el Relator Especial de Naciones Unidas para la Libertad de Opinión y Expresión (ONU), el Representante de la Organización de Seguridad y Cooperación en Europa para la Libertad de los Medios de Comunicación (OSCE) y el Relator Especial para la Libertad de Expresión de la Organización de Estados Americanos (OEA), afirmaron:

La promoción de la diversidad debe ser el objetivo primordial de la reglamentación de la radiodifusión; la diversidad implica igualdad de género en la radiodifusión e igualdad de oportunidades para el acceso de todos los segmentos de la sociedad a las ondas de radiodifusión.

Más adelante, los relatores de ONU, OEA, OSCE y la Relatora Especial sobre Libertad de Expresión y Acceso a la Información de la Comisión Africana de Derechos Humanos y de los Pueblos (CADHP) expresaron en su Declaración Conjunta sobre la Diversidad en la Radiodifusión de 2007 que: "Los diferentes tipos de medios de comunicación -comerciales, de servicios públicos y comunitarios- deben ser capaces de operar en, y tener acceso equitativo a todas las plataformas de transmisión disponibles". Luego, en 2009 la Relatoría Especial para la Libertad de Expresión a cargo de Catalina Botero elaboró los "Estándares de libertad de expresión para una radiodifusión libre e incluyente". Entre sus disposiciones el documento afirma:

En varias oportunidades, la CIDH y la Relatoría Especial han reconocido que los medios de comunicación comunitarios cumplen en nuestra región una función fundamental para el ejercicio de distintos sectores de la sociedad a la libertad de expresión y al acceso a la información. En dichos pronunciamientos han establecido que resulta necesario que los Estados los reconozcan legalmente y que se contemplen reservas de espectro para este tipo de medios, así como condiciones equitativas de acceso a las licencias que diferencien las realidades distintas de los medios privados no comerciales.

Estos estándares fueron aprobados en diciembre del mismo año por la Comisión Interamericana de Derechos Humanos.

Los propósitos de esta estrategia política deliberada, coherente, sistemática y progresiva a escala global para la incidencia en los estándares internacionales en materia de derecho -sobre la libertad de expresión y a la información, entendidos como marcos interpretativos de estos derechos, no se agotaron en sí mismos. Por el contrario, en un contexto de creciente globalización fue el camino que las radios comunitarias, populares y alternativas encontraron, 
a través de las redes en torno a las que se nuclearon, para incidir en las legislaciones latinoamericanas que consideraban ilegales -hacia las emisoras o que, en el mejor de los casos, las reconocían con restricciones. Si cada vez más las legislaciones nacionales debían adecuarse los tratados internacionales a los que los Estados nacionales se adherían, la incidencia en los marcos interpretativos de aquellos tratados se volvió una cuestión relevante para la presión sobre los propios Estados y gobiernos. La estrategia no resultó en vano: paulatinamente entrado el nuevo siglo diversos países de la región modificaron sus legislaciones en materia de radiodifusión en un sentido democratizador en concordancia, en determinada medida, con dichos estándares. Nos referimos, por ejemplo, al reconocimiento legal que las radios comunitarias, populares y alternativas lograron a través de la Ley de Servicio de Radiodifusión Comunitaria en Uruguay en 2007, la Ley de Servicios de Comunicación Audiovisual en Argentina en 2009 y la Ley Orgánica de Comunicación en Ecuador en 2013. Lo que siguió luego de estas leyes, es asunto de una indagación y reflexión que excede el propósito de este trabajo.

\section{Hacia un nuevo activismo global}

Podemos hipotetizar provisoriamente ${ }^{5}$ que el desarrollo de políticas de comunicación democratizadoras por parte de los gobiernos populistas de izquierda latinoamericanos, primeramente, y la reciente regresividad de derechos comunicacionales a partir de las nuevas políticas desplegadas por los gobiernos neoconservadores de la región, desplazaron la atención del activismo que nucleó a expertos y militantes sociales de la escena global a los territorios nacionales. Paralelamente, la emergencia de nuevas plataformas y nuevos actores de la comunicación en el contexto de despliegue de Internet y las tecnologías digitales configuran un escenario que plantea novedosos y urgentes desafíos a quienes pugnan por la democratización de las comunicaciones. ¿Cómo regular los servicios OTT desde los principios de diversidad y pluralismo? ¿Cómo garantizar federalismo y producción nacional en estas plataformas? ¿Qué implicancias regulatorias tiene la comprensión del acceso a Internet como un derecho? ¿Quiénes y cómo se controla la censura que ejercen los intermediarios de Internet como Facebook y Google? ¿Cómo regular las fake news sin restringir el derecho a la libertad de expresión? ¿Cómo limitar la concentración de las industrias culturales a las que tienden los procesos de convergencia tecnológica? ¿Cómo preservar los derechos de trabajadoras y trabajadores en tiempos de prosumidores? ¿Qué capacidad legal y qué fuerza política tienen los Estados nacionales en la regulación de los grupos económicos extranjeros dedicados a las comunicaciones?

5 Decimos hipotetizar provisoriamente pues no nos dedicaremos a demostrar esta hipótesis sino que la dejamos planteada como provocación para la reflexión política. 
Quizás la pequeña gran historia del movimiento de radios comunitarias, populares y alternativas que acabamos de relatar sea inspiradora de cara a los desafíos en tiempos digitales. Dar vida a un activismo comunicacional de escala global que articule a expertos y militantes sociales, que enrede a diversos organismos nacionales e internacionales, que construya respuestas propositivas a estos interrogantes, que renuncie a la marginalidad en tiempos aciagos y tenga profunda voluntad de incidencia, que elabore una estrategia deliberada y sostenida en el tiempo, que erija el derecho a la comunicación y la democratización de las sociedades como horizonte irrenunciable, puede ser un camino que valga la pena ensayar.

\section{Referencias bibliográficas}

ALER. (1996). Un nuevo horizonte teórico par la radio popular en Emérica Latina. Quito: ALER. ALER. (2007). La vuelta y media. Obtenido de Reflexiones alrededor del Proyecto Político Comunicativo: Recuperado de http://www.aler.org/docs/libros/vuelta_y_media.pdf

ALER Y AMARC ALC. (2009). Transformar. El camino y los andares. Buenos Aires: ALER Y AMARC ALC.

AMARC ALC. (2009). Informe sobre Diversidad y Pluralismo en la Radiodifusión en América Latina 2009. Recuperado de: http://legislaciones.amarc.org/Informe2009_Diversidad_y_Pluralismo.pdf.

AMARC ALC. (2009). Principios para un marco regulatorio democrático sobre radio y TV comunitaria. Buenos Aires: AMARC ALC.

ALER (1996). Un nuevo horizonte teórico para la radio popular en América Latina. Quito: ALER.

ALER (2007). La vuelta y media. Reflexiones alrededor del Proyecto Político Comunicativo. Recuperado de http://www.aler.org/docs/libros/vuelta y media.pdf

ALER y AMARC ALC (2009). Transformar. El camino y los andares. Buenos Aires: ALER y AMARC ALC

AMARC ALC (2009 a). Principios para un marco regulatorio democrático sobre radio y TV comunitaria. Buenos Aires: AMARC ALC.

AMARC ALC (2009 b). Informe sobre Diversidad y Pluralismo en la Radiodifusión en América Latina 2009. Recuperado de: http://legislaciones.amarc.org/Informe2009 Diversidad y Pluralismo.pdf

AMARC ALC (2010 a). Principios para garantizar la diversidad y el pluralismo en la radiodifusión y los servicios de comunicación audiovisual. Buenos Aires: AMARC ALC.

AMARC ALC (2010 b). Informe sobre Diversidad y Pluralismo en la Radiodifusión en América Latina 2010. Recuperado de: http://www.amarc.org/documents/rapports/annual reports/Informe2010.pdf

Busso, N. (2011). Derecho a la Comunicación: conceptos clave y contexto internacional. En Busso, N. \& Jaimes, D. (comp.). La cocina de la ley. El proceso de incidencia en la elaboración de la Ley de Servicios de Comunicación Audiovisual en Argentina. Buenos Aires: Buenos Aires.

Geerts, A., Van Oeyen, V. \& Villamayor, C. (2004). La radio popular y comunitaria frente al nuevo siglo: la práctica inspira. Quito: ALER y AMARC ALC.

Gerbaldo, J. (2014). Radios Comunitarias, Comunicación Popular y Ciudadanía. Disputas por 
la democratización de la palabra pública. Su tesis de maestría. El caso del Foro Argentino de Radios Comunitarias -FARCO- Argentina (1980-2013). (Tesis de Maestría). Córdoba: CEA-UNC.

Galavotti, R. \& Randazzo, D. (2001). FM de la Calle. Una historia que merece ser contada. Mendoza.

Girard, B. (1997). Pluralismo, radio e Internet. Revista Chasqui, 59, 26-28.

Gómez, G. \& Aguerre, C. (coord.) (2009). Las mordazas invisibles. Buenos Aires: AMARC ALC. Gutiérrez, H. (1997). La radio popular. Entre lo local y lo global. Revista Chasqui, 59, 29-32. Laclau, E. (2011). La razón populista. Buenos Aires: Fondo de Cultura Económica.

Laclau, E. y Mouffe, Ch. (2010). Hegemonía y estrategia socialista. Buenos Aires: Fondo de Cultura Económica.

Lamas, E. \& y Lewin, H. (1995). Aproximación a las radios de Nuevo tipo: tradición y -escenarios actuales". Revista Causas y Azares, 2, 70-86.

López Vigil, J. I. (1997). Manual urgente para radialistas apasionados. Quito: AMARC ALC y otros.

Mata, M. C. (1993). ¿Radio popular o comunitaria? Revista Chasqui, 47, 57-59.

Mata, M. C. (1994). Hacer radio es producir realidad. Revista Chasqui, 49, 44-47.

Mattelart, A. (2007). Pasado y presente de la Sociedad de la Información: entre el Nuevo Orden Mundial de la Información y la Comunicación y la Cumbre Mundial sobre la Sociedad de la Información. En Loreti, D., Mastrini, G. \& Baranchuk, M. (comp.). Participación y democracia en la Sociedad de la Información. Buenos Aires: Prometeo.

Roncagliolo, R. (1996). Libertad de expresión radiofónica. Revista Chasqui, 56, 48-51.

Villamayor, C. \& Lamas, E. (1998). Gestión de la radio comunitaria y ciudadana. Quito: FES y AMARC ALC.

\section{Declaraciones:}

Declaración de Pozo de Rosas, realizada en el marco del seminario "Democratizar el espectro radioeléctrico”. Pozo de Rosas, Venezuela, 15 de noviembre de 1996.

Declaración conjunta del Relator Especial de Naciones Unidas para la Libertad de Opinión y Expresión (ONU), el Representante de la Organización de Seguridad y Cooperación en Europa para la Libertad de los Medios de Comunicación (OSCE) y el Relator Especial para la Libertad de Expresión de la Organización de Estados Americanos (OEA). 2001.

Declaración conjunta sobre la Diversidad en la Radiodifusión del Relator Especial de Naciones Unidas para la Libertad de Opinión y Expresión (ONU), el Relator Especial para la Libertad de Expresión de la Organización de Estados Americanos (OEA), el Representante de la Organización de Seguridad y Cooperación en Europa para la Libertad de los Medios de Comunicación (OSCE) y la Relatora Especial sobre Libertad de Expresión y Acceso a la Información de la Comisión Africana de Derechos Humanos y de los Pueblos (CADHP). 2007. 
LARISA KEJVAL 